\title{
Advantage from massive parallel sequencing of RYR1 and CACNA1S in diagnostics of malignant hyperthermia susceptibility
}

\author{
Dorota Fiszer ${ }^{1,2^{*}}$, Nickla A Fisher ${ }^{2}$, Ian M Carr ${ }^{1}$, Marie-Anne Shaw ${ }^{1,2}$, Philip M Hopkins ${ }^{1,2}$ \\ From 33rd Annual Meeting of the European Malignant Hyperthermia Group (EMHG) \\ Würzburg, Germany. 15-17 May 2014
}

\section{Background}

Mutations in RYR1 (ryanodine receptor type 1) are linked to the majority of malignant hyperthermia $(\mathrm{MH})$ families (75\%) and some cases of Exertional Heat Stroke. Two CACNA1S variants associated with $\mathrm{MH}$ have been functionally characterised. Historically, because of the large size of the RYR1 gene, MH families were screened for 31 diagnostic RYR1 variants only. Methods based on PCR and Sanger sequencing were used. The new opportunity arose since next generation sequencing techniques became available. With this technique sequencing is considerably quicker therefore even long gene sequences such as $R Y R 1$ can be easily screened for variations.

\section{Material and methods}

We used Next-Generation Sequencing to look for coding sequence non-synonymous variants of $R Y R 1$ and CACNA1S. We applied two different target enrichment methods, Long-Range PCR and HaloPlex (Agilent), to be able to focus on particular regions of the genome.

\section{Results}

In our study we included a group of 80 unrelated MHS individuals who were previously screened for diagnostic mutations in $R Y R 1$ and were found negative. Another group consisted of discordant individuals, whose in vitro contracture test (IVCT) phenotype was at odds with their genotype for a familial diagnostic RYR1 mutation. The third studied cohort $(\mathrm{n}=38)$ were Exertional Heat Stroke patients, IVCT tested susceptible or normal.

\section{Conclusions}

Our results illustrate that Next Generation Sequencing provides broad genetic information quicker and at lower cost than conventional approaches. To improve understanding of malignant hyperthermia genetics, screening of susceptible individuals only for a limited number of diagnostic $R Y R 1$ variants is not sufficient. Studying the full length of RYR1 and CACNA1S coding sequence may also resolve some phenotype/genotype discordant cases. Finally, Next Generation Sequencing techniques provide opportunities for studying larger gDNA fragments or the whole genome, which may result in discovering some other genes linked to $\mathrm{MH}$.

\section{Authors' details}

${ }^{1}$ Leeds Institute of Biomedical \& Clinical Sciences, School of Medicine, University of Leeds, Leeds, LS9 7TF, UK. ${ }^{2}$ Malignant Hyperthermia Investigation Unit, St James's University Hospital, Leeds, LS9 7TF, UK.

Published: 18 August 2014 